\title{
BMJ Open Clinical and genetic associations of renal function and diabetic kidney disease in the United Arab Emirates: a cross-sectional study
}

\author{
Wael M Osman, ${ }^{1}$ Herbert F Jelinek, ${ }^{2,3}$ Guan K Tay, ${ }^{1,4,5,6}$ Ahsan H Khandoker, ${ }^{6}$ \\ Kinda Khalaf, ${ }^{6}$ Wael Almahmeed, ${ }^{7,8}$ Mohamed H Hassan, ${ }^{9}$ Habiba S Alsafar ${ }^{1,6}$
}

To cite: Osman WM, Jelinek HF, Tay GK, et al. Clinical and genetic associations of renal function and diabetic kidney disease in the United Arab Emirates: a crosssectional study. BMJ Open 2018;8:e020759. doi:10.1136/ bmjopen-2017-020759

- Prepublication history for this paper is available online. To view these files, please visit the journal online (http://dx.doi. org/10.1136/bmjopen-2017020759).

Received 26 November 2017 Revised 18 0ctober 2018 Accepted 20 November 2018

Check for updates

(c) Author(s) (or their employer(s)) 2018. Re-use permitted under CC BY-NC. No commercial re-use. See rights and permissions. Published by BMJ.

For numbered affiliations see end of article.

Correspondence to Dr Habiba S Alsafar; habiba.alsafar@ku.ac.ae

\section{ABSTRACT}

Objectives Within the Emirati population, risk factors and genetic predisposition to diabetic kidney disease (DKD) have not yet been investigated. The aim of this research was to determine potential clinical, laboratory and reported genetic loci as risk factors for DKD.

Research design and methods Four hundred and ninety unrelated Emirati nationals with type 2 diabetes mellitus (T2DM) were recruited with and without DKD, and clinical and laboratory data were obtained. Following adjustments for possible confounders, a logistic regression model was developed to test the associations of 63 single nucleotide polymorphisms (SNPs) in 43 genetic loci with DKD (145 patients with DKD and 265 without DKD). Linear regression models, adjusted for age and gender, were then used to study the genetic associations of five renal function traits, including 83 SNPs with albumin-to-creatinine ratio, 92 SNPs with vitamin D (25-OH cholecalciferol), 288 SNPs with estimated glomerular filtration rate (eGFR), 363 SNPs with serum creatinine and 73 SNPs with blood urea.

Results Patients with DKD, as compared with those without the disease, were mostly men ( $52 \%$ vs $38 \%$ for controls), older (67vs59 years) and had significant rates of hypertension and dyslipidaemia. Furthermore, patients with DKD had T2DM for a longer duration of time (16vs10 years), which in an additive manner was the single factor that significantly contributed to the development of $\mathrm{DKD}(\mathrm{p}=0.02, \mathrm{OR}=3.12,95 \% \mathrm{Cl} 1.21$ to 8.02). Among the replicated associations of the genetic loci with different renal function traits, the most notable included SHROOM3 with levels of serum creatinine, eGFR and DKD $\left(P_{\text {adjusted }}=0.04,0 R=1.46\right) ; C A S R, G C$ and CYP2R1 with vitamin D levels; as well as WDR72 with serum creatinine and eGFR levels.

Conclusions Associations were found between several genetic loci and risk markers for DKD, which may influence kidney function traits and DKD in a population of Arab ancestry.

\section{INTRODUCTION}

This overall dramatic worldwide increase in the number of people with diabetes has had a major impact on the increasing incidence and prevalence of diabetic kidney disease

\section{Strengths and limitations of this study}

- This cross-sectional study to determine the clinical, laboratory and genetic associations of diabetic kidney disease (DKD) and renal function traits in a sample of patients with type 2 diabetes mellitus was the first of its kind in a population of Arab ancestry from the United Arab Emirates.

- A limitation inherent to this study was that the analyses carried out did not include treatment modalities due to patients having multiple conditions and treatments, which made the models largely unstable and difficult to interpret.

- Current analyses of the DKD genetic association had a statistical power of $~ 57 \%$, suggesting that a larger population sample across the Middle East is required to discover novel clinical and genetic predisposition to $D K D$ in the region with better statistical power.

(DKD) as one of the most frequent complications of both types of diabetes. Globally, the prevalence of chronic kidney disease (CKD) among adults in the general population is reported to be around $10 \% .{ }^{1}$ However, $20 \%$ of adults with type 2 diabetes mellitus (T2DM) are expected to develop DKD based on estimated glomerular filtration rate (eGFR) measurements $\left(<60 \mathrm{~mL} / \mathrm{min} / 1.73 \mathrm{~m}^{2}\right)$, while the number reaches $30 \%-50 \%$ based on the urinary albumin excretion levels. ${ }^{1}$ This considerable variation is due to variances in settings, geographical area and ethnicity. ${ }^{2}$ Overall, the risk of DKD in T2DM is approximately $2 \%$ per year. ${ }^{3}$ In the Arab world, the prevalence of DKD is also highly variable $(10.8 \%-61.2 \%) \%)$ depending on the study design, population, sample selection, race, age, sex, as well as diagnostic criteria among other factors. ${ }^{4}$ Meta-analysis has shown that DKD is the leading cause of end-stage renal disease (ESRD) in the Gulf Cooperation Council (GCC) with a prevalence of $\sim 17 \% .^{5}$ Patients with ESRD have a $20 \%$ annual 
mortality rate, which is higher than the rate for many solid cancers. ${ }^{6}$ In addition to increasing the risk of cardiovascular morbidity and mortality ${ }^{78}$, DKD is reported to be the single strongest predictor of mortality in patients with diabetes, ${ }^{9}$ with a 5 -year survival in the range of $30 \% .{ }^{10}$

The current trend suggests that the prevalence of DKD will continue to increase worldwide, leading to increased morbidity and mortality and imposing significant socioeconomic burdens on global healthcare systems. ${ }^{11} \mathrm{~A}$ thorough literature search reveals that there remains a wide knowledge gap related to the understanding of risk factors and pathophysiological mechanisms associated with DKD, especially in the GCC and Middle East. Since ESRD can only be treated with highly invasive and costly procedures, such as dialysis or kidney transplantation, better knowledge of genetic, clinical and epidemiological factors associated with DKD is required to allow for timely and more effective treatment options.

In clinical practice, renal function is assessed using a number of tests that are reported to have high heritability rates. ${ }^{12}$ This indicates that genetic factors contribute significantly to interindividual variance in kidney function, and hence, to the susceptibility to CKD and related conditions. Thus far, several genetic loci have been linked to DKD, CKD and renal function traits in adults ${ }^{13-24}$ as well as children. ${ }^{25}$ However, in comparison with other diseases, including T2DM and other cardiometabolic disorders, studies of kidney disease and kidney function traits are largely insufficient and inconclusive. In spite of efforts to describe novel biomarkers for DKD, currently no tested candidates outperform albumin. A recent report by Saulnier et al suggests that three serum biomarkers (midregional-proadrenomedullin, soluble tumour necrosis factor receptor 1 and N-terminal prohormone brain natriuretic peptide) can improve risk prediction of the loss of renal function in patients with T2DM, in addition to the established risk factors for DKD such as age, sex, diabetes duration, HbA1c, blood pressure, baseline eGFR and albumin-to-creatinine ratio. ${ }^{26}$ However, issues such as whether the levels of these markers are affected by genetic variations, and whether the encoding genes contribute to DKD development and progress need further investigation.

The United Arab Emirates (UAE) is among the countries with the highest prevalence rates of T2DM, obesity and cardiovascular disease. ${ }^{27}$ AlSafar and colleagues have recently reported that approximately $80 \%$ of patients with T2DM within UAE present with at least one complication associated with T2DM, including kidney disease (approximately 6\%). ${ }^{29}$ Furthermore, there is increasing evidence suggesting that the genome structure of individuals of Arabic descent is different from individuals from other populations. ${ }^{30}$ Despite the high prevalence rate of DKD in the UAE, there have been no investigations up to date of the genetic associations of chronic kidney conditions and kidney functions, particularly as associated with T2DM. Therefore, the current work aimed to investigate the clinical and laboratory variables linked to DKD in a T2DM Emirati population and to study the associations of the reported genetic loci linked to different renal function tests in CKD and DKD.

\section{MATERIALS AND METHODS \\ Study type and subjects}

This work describes a cross-sectional study of Emirati patients from the city of Abu Dhabi. The demographic information and clinical data for the participants are presented in tables 1 and 2. Four hundred and ninety $(\mathrm{n}=490)$ patients with T2DM were included in the study,

Table 1 Baseline data of tested traits and tested SNPs

\begin{tabular}{|c|c|c|c|c|c|}
\hline Trait & $\begin{array}{l}\text { Mean } \pm S D \text { or } \\
\text { quartiles }\end{array}$ & $\begin{array}{l}\mathrm{N} \text { of subjects } \\
\text { (male/female) }\end{array}$ & $\begin{array}{l}\text { N of SNPs } \\
\text { reported* }^{*}\end{array}$ & $\mathbf{N}$ of SNPs tested ${ }^{*}$ & Covariates \\
\hline ACR (mg/mmol) & 1.05/3.9/12.7† & $115(56 / 59)$ & 331 & 83 & Age and gender. \\
\hline $\begin{array}{l}\text { eGFR }(\mathrm{ml} / \\
\left.\mathrm{min} / 1.73 \mathrm{~m}^{2}\right)\end{array}$ & $81.5 \pm 28.5$ & $395(172 / 223)$ & 1478 & 288 & Age and gender. \\
\hline $\begin{array}{l}\text { Blood urea } \\
(\mathrm{mmol} / \mathrm{L})\end{array}$ & $6.4 \pm 5.4$ & $450(263 / 214)$ & 446 & 73 & Age and gender. \\
\hline $\begin{array}{l}\text { Diabetic kidney } \\
\text { disease }\end{array}$ & & $\begin{array}{l}\text { With: } 145 . \\
\text { Without: } 265 .\end{array}$ & 288 & 63 & $\begin{array}{l}\text { Age, gender, } \\
\text { hypertension, T2DM } \\
\text { duration and eGFR } \\
\text { levels. }\end{array}$ \\
\hline
\end{tabular}

${ }^{*}$ Reported means the total number of SNPs found in the search in the literature and tested means the actual number of SNPs found from the reported SNPs and used for the analyses in this study for each corresponding trait.

†For the albumin:creatinine ratio (ACR) and creatinine analyses, summary statistics indicated by 25 th/50th/75th quartiles and not as mean \pm SD because their distributions are skewed.

eGFR, estimated glomerular filtration rate; SNPs, single nucleotide polymorphisms; T2DM, type 2 diabetes mellitus. 
Table 2 Demographic, clinical and laboratory characteristics of T2DM patients with or without kidney disease

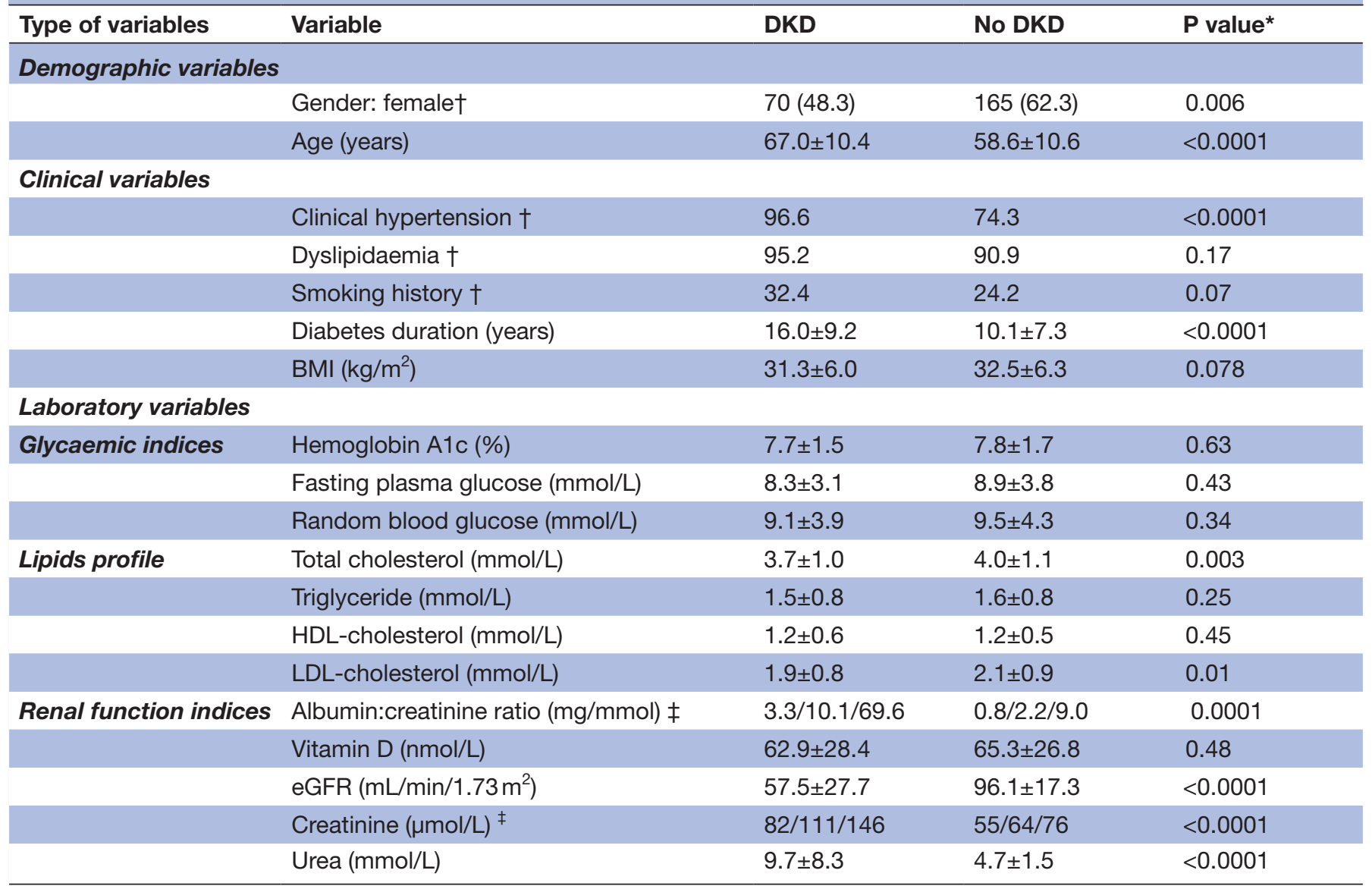

${ }^{*} \mathrm{P}$ value for continuous data, calculated using two-sided t-test except for albumin:creatinine ratio and creatinine where it is for Wilcoxon ranksum (Mann-Whitney) test. $\mathrm{P}$ value for percentage data calculated using Pearson $\chi^{2}$ test with exception of hypertension and dyslipidaemia where Fisher's exact test was used.

$\dagger$ Proportional data shown as number of positive outcome and its percentage. All remaining continuous data shown as mean \pm SD except albumin:creatinine ratio and creatinine.

\#For the albumin:creatinine ratio and creatinine analyses, summary statistics indicated by 25 th/50th/75 quartiles and not as mean \pm SD because their distributions are highly skewed.

BMI, body mass index; DKD, diabetic kidney disease; eGFR, estimated glomerular filtration rate; HDL, high-density lipoprotein; LDL, highdensity lipoprotein; T2DM, type 2 diabetes mellitus.

with 145 diagnosed with $\mathrm{DKD}$. The participants were recruited from Sheikh Khalifa Medical City and Mafraq Hospital, major tertiary hospitals in Abu Dhabi, UAE. All subjects were UAE born and of Arabian descent.

\section{Patient and public involvement}

The study was designed because T2DM, along with its multiorgan complications, is a major health challenge in the UAE with increasingly growing public interest. However, patients and the public at large were not involved in defining the research questions, analyses, interpretation or dissemination of the results.

\section{Clinical variables and laboratory data}

Various clinical and laboratory measures were collected/ assessed during the hospital visits. Blood pressure was taken on two different occasions. Hypertension was defined as systolic blood pressure $\geq 140 \mathrm{~mm} \mathrm{Hg}$, diastolic blood pressure $\geq 90 \mathrm{~mm} \mathrm{Hg}$ or if the patients were taking any antihypertensive medications. Dyslipidaemia was either reported based on clinical records of the participants or diagnosed as previously indicated. ${ }^{31}$ The presence of T2DM was confirmed by a qualified physician based on criteria outlined by the WHO. ${ }^{32}$ Trained nurses measured the height and weight of each participant using a calibrated wall-mounted stadiometer and a weight scale, respectively. Body mass index was calculated as the weight in kilograms divided by the square of the height in metres $\left(\mathrm{kg} / \mathrm{m}^{2}\right)$.

\section{Diabetic kidney disease}

DKD was defined as either decreased levels of eGFRestimated glomerular filtration rate $\left(<60 \mathrm{~mL} / \mathrm{min} / 1.73 \mathrm{~m}^{2}\right)$ with or without renal damage over a period of at least 3 months, ${ }^{33}$ or based on an albumin-to-creatinine ratio $\geq 30 \mathrm{mg} / \mathrm{g}$, or proteinuria $>500 \mathrm{mg}$ over a 24 -hour period in the setting of T2DM and/or abnormalities, as assessed by imaging or histology. ${ }^{34}$ eGFR was calculated according to CKD-EPI Creatinine Equation. ${ }^{35}$ 
Accordingly, 145 patients with TD2M were identified with DKD, while 265 were identified as disease free (tables 1 and 2). The remaining 80 patients could not be classified with or without DKD at the time of the study and were excluded from subsequent analyses.

\section{Selection of SNPS}

Genetic investigation in this study followed the candidate gene approach. ${ }^{36}$ The SNPs tested for each trait are summarised in table 1. These SNPs were selected from a recent Genome Wide Association Study (GWAS) that was intended to determine the genetic associations of T2DM in the UAE population towards establishing the Emirates Family Registry for T2DM. ${ }^{27}$ The GWAS was performed for 490 samples with T2DM and 450 healthy controls using the Infinium Omni5ExomeHuman chip (Illumina, San Diego, California, USA). To select the SNPs associated with the kidney function traits included in the study (blood urea, serum creatinine, eGFR values, albumin-to-creatinine ratio and vitamin D levels) as well as DKD, various search engines and data bases including PubMed, Google Scholar, the GWAS catalogue (https:// www.ebi.ac.uk/gwas/home), the Phenolyzer database (http://phenolyzer.wglab.org/), the infinome genome interpretation platform (https://www.infino.me/) and the GWAS Central database (http://www.gwascentral. org/) were consulted.

Our search strategy consisted of identifying reported SNPs that cleared the GWAS significance level and were found in our GWAS data. If the original signal SNP was missing from the GWAS data, we searched for a possible proxy SNP using the concept of linkage disequilibrium (LD). This typically indicates a non-random association of alleles at different genetic loci in a given population and their tendency to be inherited as a block (mathematical values $r^{2}$ and $D^{\prime}>0.8$ indicates high LD). Proxy SNPs were selected using the SNAP database for SNP Annotation and Proxy Search (http://archive.broadinstitute. org/mpg/snap/ldsearch.php).

All SNPs located within and flanking genes that have been previously reported in association with CKD and DKD were included. In total, 43 genetic loci were identified as linked to CKD, DKD or a decline in renal function. Specifically, the gene loci comprised: $A C A C B, A C E$, ACTN4, ADIPOQ ADM, AFF3, AGTR1, APOL1, CARSCNDP1, CNDP2, CPS1, CPVL, CPVL, CHN2, CYBA-ELMO1, ENPP1, ERBB4, FABP2, FRMD3, GLUT1, IRS2, MYO16, LIMK2, MCTP2, MYO16, MYH9, NCALD, NCK1, NOS3, NPHS1, NPHS2, NPPB, PLCE1, PPARY2, PVT1, RAGE, RGMA, RPS12, SFI1, SHROOM3, TMEM22, TNFRSF1A and TRPC6.

\section{Statistical modelling and analyses}

Continuous variables were presented as mean $\pm \mathrm{SD}$ or lower/median and upper quartiles where the distributions were highly skewed. Vitamin D and eGFR levels were normally distributed. However, urea levels, creatinine levels and albumin-to-creatinine ratio data were converted to normal distributions using natural $\log$ transformation. The associations of trait values or their natural logs (if transformed) were tested with SNPs using linear regression models, which included age and gender as covariates using PLINK software V.1.07 (http://zzz.bwh.harvard.edu/plink/). The same software was used for counting allele frequencies and testing the quality control (QC) variables. Any SNPs with minor allele frequency (MAF) $<0.05$, and $>5 \%$ missing genotype rate, or those that failed the Hardy-Weinberg equilibrium (HWE) test at the 0.001 were excluded. HWE is considered as an important QC test for genetic association studies and assumes that allele and genotype frequencies can be estimated. If the frequencies of the measured genotypes significantly differed from the HWE assumptions, genotyping errors among other possible factors, such as ethnic diversity and high levels of consanguinity in the population, are indicated leading to excluding the SNPs from further analyses. Association with $\mathrm{p}<0.05$ were reported, indicating the replication of previously reported associations.

Statistical analyses for all clinical and laboratory variables were performed using Stata software V.14 . For continuous data, statistical differences were assessed using two-sided t-tests for normally distributed data or the Wilcoxon rank-sum (Mann-Whitney) test for highly skewed data. The Pearson $\chi^{2}$ test was used for percentage data as well as the Fisher's exact test when the expected frequencies were less than 5 . A p value $<0.05$ was considered as significant. PLINK software was also used for testing the associations between the SNPs and DKD using a case-control logistic regression model, which included age, gender, hypertension status, T2DM duration and eGFR levels as covariates (table 1 ). The results are presented as $\mathrm{p}$ values $\left(\mathrm{P}_{\text {adjusted }}<0.05\right)$ and ORs with corresponding 95\% CIs. The same approach (patients with DKD vs patients without $\mathrm{DKD}$ ) was adopted to test the associations between the possible risk factors and the development of the DKD (table 3). However, the logistic model, which included all the associated factors listed in table 4 was validated by the Hosmer-Lemeshow goodness of fit test $(p=0.11)$ to allow for the inclusion of several covariates. Analyses between SNPs and renal function traits were conducted in PLINK using linear regression models that included age and gender as covariates. The results were presented as beta coefficients (regression coefficients for the linear regression model, calculated based on the minor allele), standard errors and $p$ values.

As this was a replication study, we reported all $\mathrm{p}$ values $<0.05$, suggesting possible replications. However, using a Bonferroni correction for multiple testing, the $p$ values of some of the models with statistically significant associations included $\mathrm{p}<0.00079$ for the DKD associations, $\mathrm{p}<0.0006$ for the ACR, $\mathrm{p}<0.0005$ for the vitamin $\mathrm{D}, \mathrm{p}<0.00017$ for the eGFR, $\mathrm{p}<0.00019$ for the creatinine and $\mathrm{p}<0.00068$ for associations with urea. 
Table 3 Risk factors for the development of kidney disease in patients with T2DM from the UAE

\begin{tabular}{llll}
\hline Covariate & OR & $\mathbf{9 5 \%}$ Cl & P values \\
\hline Age & 0.99 & 0.96 to 1.04 & 0.80 \\
\hline Gender & 0.90 & 0.34 to 2.36 & 0.83 \\
\hline BMI & 1.02 & 0.97 to 1.08 & 0.49 \\
\hline Hypertension & 2.00 & 0.60 to 6.74 & 0.26 \\
\hline HbA1c & 0.86 & 0.69 to 1.07 & 0.19 \\
\hline Cholesterol & 0.59 & 0.19 to 1.76 & 0.34 \\
\hline Triglyceride & 1.09 & 0.62 to 1.94 & 0.76 \\
\hline HDL-cholesterol & 1.25 & 0.64 to 2.45 & 0.52 \\
\hline LDL-cholesterol & 1.38 & 0.42 to 4.57 & 0.60 \\
\hline Smoking history & 0.80 & 0.34 to 1.87 & 0.61 \\
\hline Creatinine & 1.03 & 1.00 to 1.06 & 0.03 \\
\hline Urea & 1.13 & 0.92 to 1.38 & 0.26 \\
\hline eGFR & 0.97 & 0.94 to 1.00 & 0.09 \\
\hline
\end{tabular}

Diabetes

duration (years)*

\begin{tabular}{llll}
\hline-10 & 0.75 & 0.26 to 2.16 & 0.59 \\
$11-15$ & 1.49 & 0.56 to 3.91 & 0.42 \\
$16-20$ & 3.35 & 1.22 to 9.23 & 0.02 \\
$>20$ & 3.12 & 1.21 to 8.02 & $\mathbf{0 . 0 2}$ \\
\hline
\end{tabular}

*Diabetes duration reference is duration $\leq 5$ years

$\mathrm{BMI}$, body mass index; eGFR, estimated glomerular filtration rate; HDL, high-density lipoprotein; LDL, low-density lipoprotein; T2DM, type 2 diabetes mellitus; UAE, United Arab Emirates.

\section{Statistical power considerations}

The current study had a power $\sim 57 \%$. This is based on the current sample size (145 with DKD vs 265 without DKD), prevalence of DKD $\sim 20 \%$ (depending on reference \#2), genotype risk $(\mathrm{OR})=1.5$, significance level of 0.0008 (following the correction of multiple testing using the Bonferroni correction), disease allele frequency $=0.5$ and multiplicative model. Calculations were verified using the Genetic Association Study Power Calculator (http://csg. sph.umich.edu//abecasis/cats/gas_power_calculator/ index.html).

\section{Ethical considerations}

Each patient agreed to take part in this study and provided an informed signed consent after a brief session to explain the aims and methods. The study conformed to the ethical principles outlined in the Declaration of Helsinki.

\section{RESULTS}

\section{Baseline data of kidney function associated traits and SNPs} selection

Of the 490 patients with T2DM that were recruited for this study, 115 patients were tested for genetic associations with the albumin-to-creatinine ratio, 328 for vitamin D levels (measured as 25-OH cholecalciferol), 395 for
eGFR levels, 474 for serum creatinine levels and 450 for blood urea levels. Among the 490 patients, 410 had clear classifications for the diagnosis of kidney disease (145 patients with DKD and 265 without DKD) according to the adopted diagnostic criteria (references \#33 and \#34) and/or patient medical records. The possible covariates that may affect the genetic associations for trait analyses are summarised in table 1 .

\section{Clinical and laboratory characteristics of patients with and without DKD}

Both patient groups (with or without kidney disease) had poor glycaemic control with blood glucose levels above $8 \mathrm{mmol} / \mathrm{L}$. A comparison of patients with DKD to those without DKD indicated that the majority of patients with DKD were men (52\% vs $38 \%$ for controls), older in age (67 vs 59 years), had significant rates of comorbidities, such as hypertension and dyslipidaemia, and had a longer T2DM duration (16 vs 10 years). A clear decline in renal function indices was also observed in patients with DKD compared with those without DKD, as indicated by the significantly higher rates of ACR, urea and creatinine, and significantly lower eGFR (table 2). However, patients with DKD tended to have lower low-density lipoprotein-cholesterol results as compared with those without DKD, which suggests that these patients were more likely to have received intensive statin therapy (table 2 ).

\section{Factors associated with developing DKD in Emirati patients with T2DM}

Table 3 shows that T2DM duration was the single factor that significantly contributed to the development of DKD. Increased risk for DKD was significantly associated with the increasing duration of T2DM, cumulatively, up to 20 years of duration. At a T2DM duration $\geq 20$ years, the DKD risk stabilised at approximately 3.12 times higher as compared with patients with duration $\leq 5$ years $(\mathrm{p}=0.02$, $95 \%$ CI 1.21 to 8.02). Although the levels of serum creatinine indicated a significant difference between no DKD and DKD patients $(\mathrm{p}=0.03)$, the OR of 1.03 showed no increased risk (table 3 ).

\section{Genetic associations of renal function associated traits in Emirati patients}

The results of genetic associations for each tested renal function trait are shown in table 4. In summary, no association passed the Bonferroni correction for multiple testing. However, we report here the most suggestive associations which point to replications of previous reports.

For blood urea, the best observed association was with rs11868441 in breast carcinoma-amplified sequence 3 (BCAS3) (effect size: $-0.038 \log$ per allele $\mathrm{A}, \mathrm{p}=0.014$ ), followed by multiple SNPs in the R-Spondin 3 (RSPO3) gene.

For serum creatinine, two SNPs (rs6999484 and rs1705699) in the intergenic region between STC1 and ADAM28 showed the best associations with similar effect sizes $(0.03 \log$ per one copy of the corresponding minor 
Table 4 Results of genetic association analyses of different renal function indices

\begin{tabular}{|c|c|c|c|c|c|c|c|}
\hline SNP & Chr: BP & Gene & A1/A2* & MAF_\% & Beta† & SE & $P$ value \\
\hline \multicolumn{8}{|l|}{ Blood urea } \\
\hline rs11868441 & 17: 59239221 & $B C A S 3$ & $\mathrm{~A} / \mathrm{G}$ & 30.1 & -0.038 & 0.015 & 0.014 \\
\hline rs4644087 & 6: 127481154 & RSPO3 & $\mathrm{C} / \mathrm{A}$ & 46.8 & 0.030 & 0.014 & 0.03 \\
\hline rs4382293 & 6: 127475433 & RSPO3 & $\mathrm{G} / \mathrm{A}$ & 47.0 & 0.030 & 0.014 & 0.03 \\
\hline rs2489629 & 6: 127476717 & RSPO3 & $\mathrm{G} / \mathrm{A}$ & 44.9 & -0.029 & 0.014 & 0.039 \\
\hline rs6999484 & 8: 23728271 & STC1-ADAM28 & $\mathrm{A} / \mathrm{G}$ & 23.8 & 0.033 & 0.011 & 0.003 \\
\hline rs1705699 & 8: 23781453 & STC1-ADAM28 & $\mathrm{G} / \mathrm{A}$ & 24.2 & 0.031 & 0.011 & 0.005 \\
\hline rs2828785 & 21: 25437505 & - & $\mathrm{A} / \mathrm{G}$ & 19.9 & -0.030 & 0.011 & 0.008 \\
\hline rs11227279 & 11: 65495211 & KRT8P26-AP5B1 & $\mathrm{A} / \mathrm{G}$ & 28.4 & -0.024 & 0.010 & 0.019 \\
\hline rs7785065 & 7: 32915204 & KBTBD2 & $\mathrm{A} / \mathrm{C}$ & 43.5 & 0.022 & 0.010 & 0.022 \\
\hline \multicolumn{8}{|c|}{$\begin{array}{l}\text { Estimated glomerular } \\
\text { filtration rate }\end{array}$} \\
\hline rs2168785 & $17: 37407135$ & MED1 & $\mathrm{G} / \mathrm{A}$ & 28.2 & -4.299 & 1.754 & 0.015 \\
\hline rs 12452509 & 17: 37574722 & MED1 & $\mathrm{G} / \mathrm{A}$ & 28.1 & -4.232 & 1.757 & 0.017 \\
\hline rs4776168 & 15: 53936907 & WDR72 & $\mathrm{G} / \mathrm{A}$ & 10.8 & 5.828 & 2.699 & 0.031 \\
\hline rs10518733 & 15: 53940307 & WDR72 & $\mathrm{C} / \mathrm{A}$ & 10.8 & 5.828 & 2.699 & 0.031 \\
\hline rs7541937 & 1: 35341982 & DLGAP3 & $\mathrm{C} / \mathrm{A}$ & 44.0 & 3.590 & 1.693 & 0.035 \\
\hline rs10032549 & 4: 77398015 & SHROOM3 & $\mathrm{G} / \mathrm{A}$ & 32.2 & -3.655 & 1.750 & 0.037 \\
\hline rs2484639 & 1: 243462367 & SDCCAG8 & $\mathrm{A} / \mathrm{G}$ & 43.5 & 3.378 & 1.656 & 0.042 \\
\hline \multicolumn{8}{|c|}{$\begin{array}{l}\text { Vitamin } \mathrm{D}(25-\mathrm{OH} \\
\text { cholecalciferol) }\end{array}$} \\
\hline rs4528660 & 18: 3043516 & LPIN2-MYOM1 & $G / A$ & 17.8 & -0.323 & 0.144 & 0.027 \\
\hline
\end{tabular}

${ }^{*} \mathrm{~A} 1 / \mathrm{A} 2:$ minor to major alleles.

†Beta: regression coefficient for the linear regression model, calculated based on A1 (the minor allele).

BP, base pair position; Chr, chromosome; MAF, minor allele frequency; SNP, single nucleotide polymorphism.

allele), followed by SNP rs2828785 ( $\mathrm{p}=0.008$, effect size $=-0.030 \log$ per one copy of allele A), which is located in the non-coding gene area on chromosome 21. In addition, multiple genetic areas were also indicated, although with less significant associations.

eGFR levels were significantly associated with SNPs within the MED1 gene, rs2168785 with effect size of -4.299 per allele $G$ and rs12452509 with effect size of -4.232 per allele $\mathrm{G}$ and $\mathrm{p}=0.015$ and 0.017 , respectively. In addition, the SNPs in two genetic regions, WDR72 and SHROOM3, which are associated with serum creatinine levels, were also associated with eGFR levels, indicating a strong link to renal function.

Vitamin D levels were associated with three genetic regions including $\mathrm{rs} 1801725$ in CARS (effect size: -6.923 per allele $\mathrm{A}, \mathrm{p}=0.0078$ ), rs1155563 in $G C$ (effect size: $-6.951, \mathrm{p}=0.0081$ ) and two SNPs, rs12794714 and rs10500804, in cytochrome P450 family 2 subfamily $\mathrm{R}$ member 1 (CYP2R1) with effect sizes of approximately -5.0 and $p$ values of 0.015 and 0.016 , respectively.

One SNP, rs4528660, which is located in the intergenic region between LPIN2 and MYOM1 (effect size: -0.323 log per allele $A, p=0.027$ ) was strongly associated with the albumin-to-creatinine ratio.

These data demonstrated that renal function traits are linked to several loci in the UAE population and that some loci (eg, WDR27 and SHROOM3) are linked to more than one trait.

\section{Genetic associations of DKD in Emirati patients}

Sixty-three SNPs in 43 genetic loci, which have previously been linked to CKD or DKD, were included in our genetic analyses of DKD (145 patients with DKD versus 265 without $\mathrm{DKD})$. A logistic regression model including 
Table 5 Association between SNPs linked to CKD and DKD patients from the UAE

\begin{tabular}{|c|c|c|c|c|c|c|c|c|}
\hline \multirow[b]{2}{*}{ SNP } & \multirow[b]{2}{*}{ Chr: BP } & \multirow[b]{2}{*}{ Gene } & \multirow[b]{2}{*}{ A1/A2* } & \multicolumn{2}{|l|}{ MAF } & \multirow[b]{2}{*}{$\mathbf{P}_{\text {unadjusted }}$} & \multirow[b]{2}{*}{$\mathbf{P}_{\text {adjusted }}$} & \multirow[b]{2}{*}{ OR $(95 \% \mathrm{Cl})$} \\
\hline & & & & $D K D(\mathrm{n}=145)$ & No $D K D(\mathrm{n}=265)$ & & & \\
\hline rs7422339 & 2: 211540507 & CPS1 & $\mathrm{A} / \mathrm{C}$ & $35.8 \%$ & $27.9 \%$ & 0.019 & 0.20 & 1.25 (0.89 to 1.75$)$ \\
\hline
\end{tabular}

${ }^{*}$ A1/A2: minor to major alleles.

BP, base pair position; Chr, chromosome; CKD, chronic kidney disease; CPS1, carbamoyl-phosphate synthase; DKD, diabetic kidney disease; MAF, minor allele frequency; SHROOM3, shroom family member 3; SNPs, single nucleotide polymorphisms; UAE, United Arab Emirates.

five possible covariates that may affect the development of kidney disease was applied (table 1). As depicted in table 5 , the unadjusted analyses indicates two associations in CPS1 (rs7422339, $\mathrm{p}=0.019$ ) and SHROOM3 (rs4859682, $\mathrm{p}=0.024$ ), respectively. Following the adjustment for possible covariates, only the SHROOM3 rs4859682 remained significant $(\mathrm{p}=0.04, \mathrm{OR}=1.46)$. This confirms that $S H R O O M 3$ is a string risk locus for $\mathrm{DKD}$, considering similar associations with serum creatinine and eGFR levels.

\section{DISCUSSION}

A combination of environmental and clinical factors in genetically predisposed individuals have been suggested to be involved in DKD, including persistent hyperglycaemia, arterial hypertension and/or dyslipidaemia. ${ }^{37}$ In addition, familial aggregation of nephropathy in T2DM has been reported in several populations. ${ }^{38}$ Therefore, understanding the complex multifactorial interactions between genetic, clinical and traditional kidney disease risk factors can provide insight into novel drugs and treatment strategies for DKD towards reducing the likelihood of developing ESRD. In this study, we investigated whether the genetic markers that correspond to DKD and renal function traits reported for different populations are similar to the Arab population.

The current Emirati population sample indicated that most patients with T2DM who developed DKD were males, about 10 years older, had more frequent comorbidities, specifically hypertension and showed a marked decline in their renal function profiles, as compared with those who did not develop the disease. However, patients with T2DM who did not develop DKD still had higher rates of comorbidities and poor diabetic control, in agreement with our previous results. ${ }^{29}$ We also found that the duration of diabetes was the single factor that significantly contributed to the development of kidney disease. Specifically, the risk of developing DKD became significant when the duration of T2DM reached the 15-year mark. This is in alignment with previous reports, which suggest that patients with T2DM who do not develop signs of kidney disease by 15 years' duration of diabetes seem to be protected, most likely due to genetic factors. ${ }^{39}$

The most notable finding of this study was the association of shroom family member 3 (SHROOM3) with serum creatinine, eGFR and DKD. The minor allele for the SNP rs4859682 (A) was observed to increase the serum creatinine (0.021 log increase per one copy) and also to increase the risk for DKD (OR=1.46). SHROOM3 was first reported to be associated with eGFR levels in patients with DKD,${ }^{13}$ then with serum creatinine ${ }^{40}$ and serum magnesium levels. ${ }^{41}$ This association was further replicated in different ethnicities. ${ }^{19} 42$ The SHROOM3 gene product is expressed in the human kidney and is reported to play an important role in epithelial cell shape regulation, ${ }^{43}$ as well as the maintenance of the glomerular filtration barrier integrity. ${ }^{44}$ Defective Shroom3 protein leads to decreased actin organisation and affects the mechanical characteristics and integrity of the glomerular podocyte resulting in thinning of the glomerular filtration membrane. ${ }^{44}$ Additionally, Shroom3 heterozygous $\left(\right.$ Shroom $3^{G t /+}$ ) mice showed developmental irregularities that manifested as adult-onset glomerulosclerosis and proteinuria. ${ }^{45}$ Furthermore, genetic variants (such as the intronic variant rs17319721) were found to contribute to kidney allograft injury and the development of fibrosis through a mechanism involving transforming growth factor beta (TGF- $\beta$ ) signalling. ${ }^{46}$ Although the variant rs17319721 was not found in our dataset, it is highly linked to the SNP rs $4859682\left(r^{2}=0.85, D^{\prime}=1\right)$, which was reported in this study to increase the risk for DKD and affect the levels of serum creatinine. Overall, this suggests that SHROOM3 may be considered as a multiethnic risk gene for DKD and associated kidney function traits in various populations, including the Arabs who inhabit the UAE.

Similarly, the two loci, WD repeat domain 72 (WDR72), associated with eGFR and serum creatinine levels), and $B C A S 3$, associated with blood urea levels), are also wellknown transethnic renal function traits loci. ${ }^{47}$ WDR72, in particular, has been well studied in association with kidney function traits and pathologies. For instance, WDR72 has been reported to be associated with creatinine production or secretion, ${ }^{48}$ as well as signal transduction, cell cycle regulation and vesicular trafficking that affects podocyte activity, reduced eGFR and progression of CKD. ${ }^{49}$ In addition, $\mathrm{RSPO} 3$ has been previously reported to be associated with blood urea nitrogen concentration, in line with the results of the current study. ${ }^{42}$ The association of rs 4528660 near MYOM1 (Myomesin 1) with the albumin-to-creatinine ratio is also in agreement with previous work, which links this locus to albuminuria in patients with diabetes. ${ }^{50}$ The current analyses also replicated the associations of calcium-sensing receptor (CASR), group-specific component $(G C)$ (vitamin D-binding protein, also known 
as GC-globulin) and CYP2R1 with levels of vitamin D. ${ }^{51}$ These genes encode proteins that are involved in vitamin $\mathrm{D}$ function, including activation by hydroxylation (CYP2R1), transportation (GC) and serum calcium level sensoring (CASR) ${ }^{51}$ They have also been associated with calcium-vitamin D physiology and pathology, such as serum calcium levels, familial hypocalciuric hypercalcaemia, tertiary hyperparathyroidism and vitamin D deficiency presenting as rickets (see OMIM entries: CARS: 601199; CYP2R1: 608713; and GC: 139200). For instance, CASR protein is expressed in the kidney among other tissue and regulates ion metabolism including calcium and magnesium. Mutations in CASR lead to abnormalities in the regulation of the parathyroid gland and renal function causing hypercalcaemia and increased blood pressure, which in turn may affect kidney function. ${ }^{52}$ Similarly, GC proteins bind actin and work as actin scavengers (as such GC may play a role in podocyte integrity), as well as a binding site for vitamin D. The GC protein is the precursor to the Gc-macrophage activating factor, a macrophage activator and suggests, together with vitamin D, that GC has an important role in the immune function and pathogenesis of CKD. ${ }^{53}$ Vitamin D is hydroxylated at the C25 position by specific hydroxylase coded by the CYP2R1 gene to 25-hydroxyvitamin $\mathrm{D}$, which is the main circulating form of vitamin $\mathrm{D}$. The low levels of vitamin D observed in CKD, due to reduced CYP2R1 production by the liver or due to a mutation in the gene, disturb calcium balance and lead to hyperparathyroidism. Conversely, the loss of renal protection caused by vitamin $\mathrm{D}$ and the increase in the renin-angiotensin pathway leads to hypertension that further advances kidney disease ${ }^{54}$ Furthermore, these genes have recently been shown to influence the outcome of vitamin $\mathrm{D}_{3}$ supplementation which, in the Arab context, is an important finding of our study. ${ }^{55}$

In summary, this work presents the first study to investigate the clinical and genetic factors influencing kidney function traits and DKD in a population of Arab ancestry. The results demonstrate that the duration of T2DM is the single most important risk factor for DKD development in patients with T2DM in the UAE. Our study highlights that several genetic loci, which have been previously linked to renal function associated traits, are shared between diverse ethnic groups. As such, we have replicated previous findings of the association of SHROOM3 with DKD. The logistic analyses performed here did not include treatment modalities since most of the patients had multiple conditions and underwent multiple treatments, which makes logistic regression largely unstable and difficult to interpret. A larger population sample across the Middle East is now being considered to confirm the extent of the shared genetic predisposition reported in the current study. Considering the high prevalence of T2DM in this population and the recent evidence of genomic structure variations among different ethnic groups, more genetic-driven population studies are warranted towards effective genetically guided personalised medicine.

\section{Author affiliations}

${ }^{1}$ Center of Biotechnology, Khalifa University, Abu Dhabi, United Arab Emirates ${ }^{2}$ School of Community Health, Charles Sturt University, Albury, New South Wales, Australia

${ }^{3}$ Clinical Medicine, Macquarie University, Sydney, New South Wales, Australia ${ }^{4}$ School of Health and Medical Sciences, Edith Cowan University, Joondalup, Western Australia, Australia

${ }^{5}$ School of Psychiatry and Clinical Neurosciences, University of Western Australia, Western Australia, Australia

${ }^{6}$ Biomedical Engineering, Khalifa University, Abu Dhabi, United Arab Emirates ${ }^{7}$ Institute of Cardiac Science, Sheikh Khalifa Medical City, Abu Dhabi, United Arab Emirates

${ }^{8} \mathrm{Heart}$ and Vascular Institute, Cleveland Clinic, Abu Dhabi, United Arab Emirates ${ }^{9}$ Medical Institute, Sheikh Khalifa Medical City, Abu Dhabi, United Arab Emirates

Acknowledgements We acknowledge the patients who volunteered to make this study possible. We also acknowledge patient care advisers for their roles in this study.

Contributors HSA obtained the funding for this study. WMO, HSA and HFJ designed the study. WMO analyzed the data and prepared the manuscript. HFJ, GKT, AHK, and KK provided critical revision of the manuscript, contributed to writing the discussion, and writing the revisionof the manuscript. WA and $\mathrm{MHH}$ carried out the patient recruitment process and acquired the clinical data. All authors gave final approval of the version to be published.

Funding This study was supported by research incentive funds from Khalifa University Internal Research Fund Level 2 granted to HSA.

Competing interests None declared.

Patient consent for publication Obtained.

Ethics approval The study was approved by the Institutional Ethics Committees of both local hospitals (REC-04062014 and R292, respectively).

Provenance and peer review Not commissioned; externally peer reviewed. Data sharing statement № additional data are available.

Open access This is an open access article distributed in accordance with the Creative Commons Attribution Non Commercial (CC BY-NC 4.0) license, which permits others to distribute, remix, adapt, build upon this work non-commercially, and license their derivative works on different terms, provided the original work is properly cited, appropriate credit is given, any changes made indicated, and the use is non-commercial. See: http://creativecommons.org/licenses/by-nc/4.0/.

\section{REFERENCES}

1. Eckardt KU, Coresh J, Devuyst O, et al. Evolving importance of kidney disease: from subspecialty to global health burden. Lancet 2013;382:158-69.

2. Thomas MC, Brownlee M, Susztak K, et al. Diabetic kidney disease. Nat Rev Dis Primers 2015;1:15018.

3. Gross JL, de Azevedo MJ, Silveiro SP, et al. Diabetic nephropathy: diagnosis, prevention, and treatment. Diabetes Care 2005;28:164-76.

4. Aldukhayel A. Prevalence of diabetic nephropathy among Type 2 diabetic patients in some of the Arab countries. Int $\mathrm{J}$ Health Sci 2017;11:1.

5. Hassanien AA, Al-Shaikh F, Vamos EP, et al. Epidemiology of endstage renal disease in the countries of the Gulf Cooperation Council: a systematic review. JRSM Short Rep 2012;3:1-21.

6. Reidy K, Kang HM, Hostetter T, et al. Molecular mechanisms of diabetic kidney disease. J Clin Invest 2014;124:2333-40.

7. Matsushita K, Coresh J, Sang Y, et al. Estimated glomerular filtration rate and albuminuria for prediction of cardiovascular outcomes: a collaborative meta-analysis of individual participant data. Lancet Diabetes Endocrinol 2015;3:514-25.

8. Gansevoort RT, Correa-Rotter R, Hemmelgarn BR, et al. Chronic kidney disease and cardiovascular risk: epidemiology, mechanisms, and prevention. The Lancet 2013;382:339-52.

9. Hsu CY, Vittinghoff E, Lin F, et al. The incidence of end-stage renal disease is increasing faster than the prevalence of chronic renal insufficiency. Ann Intern Med 2004;141:95-101.

10. Collins AJ, Foley RN, Chavers B, et al. 'United States renal data system 2011 annual data report: atlas of chronic kidney disease \& end-stage renal disease in the United States. Am J Kidney Dis 2012;59:A7. 
11. de Boer IH, Rue TC, Hall YN, et al. Temporal trends in the prevalence of diabetic kidney disease in the United States. JAMA 2011;305:2532-9.

12. Fox CS, Yang $Q$, Cupples LA, et al. Genomewide linkage analysis to serum creatinine, GFR, and creatinine clearance in a communitybased population: the Framingham Heart Study. J Am Soc Nephrol 2004;15:2457-61.

13. Köttgen A, Glazer NL, Dehghan A, et al. Multiple loci associated with indices of renal function and chronic kidney disease. Nat Genet 2009;41:712-7.

14. Köttgen A, Pattaro C, Böger CA, et al. New loci associated with kidney function and chronic kidney disease. Nat Genet 2010;42:376-84.

15. Gudbjartsson DF, Holm H, Indridason OS, et al. Association of variants at UMOD with chronic kidney disease and kidney stonesrole of age and comorbid diseases. PLoS Genet 2010;6:e1001039.

16. McDonough CW, Palmer ND, Hicks PJ, et al. A genome-wide association study for diabetic nephropathy genes in African Americans. Kidney Int 2011;79:563-72.

17. Kao WH, Klag MJ, Meoni LA, et al. MYH9 is associated with nondiabetic end-stage renal disease in African Americans. Nat Genet 2008;40:1185-92.

18. Genovese G, Friedman DJ, Ross MD, et al. Association of trypanolytic ApoL1 variants with kidney disease in African Americans. Science 2010;329:841-5.

19. Chambers JC, Zhang W, Lord GM, et al. Genetic loci influencing kidney function and chronic kidney disease. Nat Genet 2010;42:373-5.

20. Okada Y, Sim X, Go MJ, Mj G, et al. Meta-analysis identifies multiple loci associated with kidney function-related traits in east Asian populations. Nat Genet 2012;44:904-9.

21. Pattaro C, Köttgen A, Teumer A, et al. Genome-wide association and functional follow-up reveals new loci for kidney function. PLoS Genet 2012;8:e1002584.

22. Yamada $\mathrm{Y}, \mathrm{Nishida} \mathrm{T}$, Ichihara $\mathrm{S}$, et al. Identification of chromosome $3 q 28$ and ALPK1 as susceptibility loci for chronic kidney disease in Japanese individuals by a genome-wide association study. $\mathrm{J}$ Med Genet 2013;50:jmedgenet-2013-101518.

23. Pattaro C, Teumer A, Gorski M, et al. Genetic associations at 53 loci highlight cell types and biological pathways relevant for kidney function. Nat Commun 2016;7:10023.

24. Palmer ND, Ng MC, Hicks PJ, et al. Evaluation of candidate nephropathy susceptibility genes in a genome-wide association study of African American diabetic kidney disease. PLoS One 2014;9:e88273.

25. Wuttke M, Wong CS, Wühl E, et al. Genetic loci associated with renal function measures and chronic kidney disease in children: the pediatric investigation for genetic factors linked with renal progression consortium. Nephrol Dial Transplant 2016;31:262-9.

26. Saulnier PJ, Gand E, Velho G, et al. Association of circulating biomarkers (adrenomedullin, tnfr1, and nt-probnp) with renal function decline in patients with type 2 diabetes: a french prospective cohort. Diabetes Care 2017;40:367-74.

27. Alsafar H, Jama-Alol KA, Hassoun AAK, et al. The prevalence of type 2 diabetes mellitus in the united arab emirates: justification for the establishment of the emirates family registry. Int J Diabetes Dev Ctries 2012;32:25-32.

28. Hajat C, Harrison O, Al Siksek Z. Weqaya: a population-wide cardiovascular screening program in Abu Dhabi, United Arab Emirates. Am J Public Health 2012;102:909-14.

29. Jelinek HF, Osman WM, Khandoker AH, et al. Clinical profiles, comorbidities and complications of type 2 diabetes mellitus in patients from United Arab Emirates. BMJ Open Diabetes Res Care 2017;5:e000427.

30. Teebi AS. Introduction: genetic diversity among arabs. Genetic disorders among Arab populations: Springer 2010:3-34.

31. Mooradian AD. Dyslipidemia in type 2 diabetes mellitus. Nat Clin Pract Endocrinol Metab 2009;5:150-9.

32. James PA, Oparil S, Carter BL, et al. 2014 evidence-based guideline for the management of high blood pressure in adults: report from the panel members appointed to the Eighth Joint National Committee (JNC 8). JAMA 2014;311:507-20.
33. Levey AS, Eckardt K-U, Tsukamoto Y, et al. Definition and classification of chronic kidney disease: A position statement from Kidney Disease: Improving Global Outcomes (KDIGO). Kidney Int 2005;67:2089-100.

34. Andrassy KM. Comments on 'KDIGO 2012 clinical practice guideline for the evaluation and management of chronic kidney disease'. Kidney Int 2013;84:622-3.

35. Levey AS, Stevens LA, Schmid CH, et al. A new equation to estimate glomerular filtration rate. Ann Intern Med 2009;150:604-12.

36. Kwon JM, Goate AM. The candidate gene approach. Alcohol Res Health 2000;24:164-8.

37. Murussi M, Coester A, Gross JL, et al. Diabetic nephropathy in type 2 diabetes mellitus: risk factors and prevention. Arquivos Brasileiros de Endocrinologia \& Metabologia 2003;47:207-19.

38. Palmer ND, Freedman BI. Insights into the genetic architecture of diabetic nephropathy. Curr Diab Rep 2012;12:423-31.

39. Krolewski AS. Genetics of diabetic nephropathy: Evidence for major and minor gene effects. Kidney Int 1999;55:1582-96.

40. Pattaro C, De Grandi A, Vitart V, et al. A meta-analysis of genomewide data from five European isolates reveals an association of COL22A1, SYT1, and GABRR2with serum creatinine level. BMC Med Genet 2010;11:41.

41. Meyer TE, Verwoert GC, Hwang S-J, et al. Genome-wide association studies of serum magnesium, potassium, and sodium concentrations identify six loci influencing serum magnesium levels. PLoS Genet 2010;6:e1001045.

42. Okada Y, Sim X, Go MJ, et al. Meta-analysis identifies multiple loci associated with kidney function-related traits in east Asian populations. Nat Genet 2012;44:904-9.

43. Nishimura T, Takeichi M. Shroom3-mediated recruitment of Rho kinases to the apical cell junctions regulates epithelial and neuroepithelial planar remodeling. Development 2008;135:1493-502.

44. Yeo NC, O'Meara CC, Bonomo JA, et al. Shroom3 contributes to the maintenance of the glomerular filtration barrier integrity. Genome Res 2015;25:57-65.

45. Khalili H, Sull A, Sarin S, et al. Developmental origins for kidney disease due to shroom3 Deficiency. Journal of the American Society of Nephrology 2016;27:2965-73.

46. Menon MC, Chuang PY, Li Z, et al. Intronic locus determines SHROOM3 expression and potentiates renal allograft fibrosis. J Clin Invest 2015;125:208-21.

47. Mahajan A, Rodan AR, Le TH, et al. Trans-ethnic fine mapping highlights kidney-function genes linked to salt sensitivity. Am J Hum Genet 2016;99:636-46.

48. O'Seaghdha CM, Fox CS. Genome-wide association studies of chronic kidney disease: what have we learned? Nat Rev Nephrol 2012;8:89-99.

49. Good MC, Zalatan JG, Lim WA. Scaffold proteins: hubs for controlling the flow of cellular information. Science 2011;332:680-6.

50. Teumer A, Tin A, Sorice R, et al. Genome-wide association studies identify genetic loci associated with albuminuria in diabetes. Diabetes 2016;65:803-17.

51. Wang TJ, Zhang F, Richards JB, et al. Common genetic determinants of vitamin $D$ insufficiency: a genome-wide association study. The Lancet 2010;376:180-8.

52. Riccardi D, Brown EM. Physiology and pathophysiology of the calcium-sensing receptor in the kidney. Am J Physiol Renal Physiol 2010;298:F485-F499.

53. Thyer L, Ward E, Smith R, et al. A novel role for a major component of the vitamin $d$ axis: vitamin $d$ binding protein-derived macrophage activating factor induces human breast cancer cell apoptosis through stimulation of macrophages. Nutrients 2013:5:2577-89.

54. Jean G, Souberbielle J, Chazot C. Vitamin D in chronic kidney disease and dialysis patients. Nutrients 2017;9:328.

55. Barry EL, Rees JR, Peacock JL, et al. Genetic variants in CYP2R1, CYP24A1, and VDR modify the efficacy of vitamin D3 supplementation for increasing serum 25-hydroxyvitamin $D$ levels in a randomized controlled trial. J Clin Endocrinol Metab 2014;99:E213 3-E2137. 